\title{
La incursión del Antropoceno en el sur del planeta
}

\author{
VIRGINIA GARCÍA ACOSTA
}

\author{
El Antropoceno es el signo de nuestra \\ potencia, pero también de nuestra \\ impotencia [...], es una toma de \\ conciencia esencial para comprender \\ lo que nos pasa. Porque lo que nos \\ pasa no es una crisis ambiental, es una \\ revolución geológica de origen humano. \\ Bonneuil y Fressoz (2013: 9-10).
}

The Incursion of the Anthropocene Discussion to the South

VIRGINIA GARCÍA ACOSTA Centro de Investigaciones y Estudios Superiores en Antropología Social-Ciudad de México, Ciudad de México, México vgarciaa@ciesas.edu.mx

Desacatos 54 , mayo-agosto 2017, pp. 8-15

na nueva época geológica marcada por el hombre está en proceso. ${ }^{2}$ Se le ha denominado Antropoceno. Se trata de una hipótesis científica basada en la suposición de que, al igual que el clima, la biodiversidad, los mares, los océanos y la tierra misma, la humanidad

"L'Anthropocène, c'est le signe de notre puissance, mais aussi de notre impuissance [...], est une prise de conscience essentielle pour comprendre ce que nous arrive. Car ce qui nous arrive n'est pas une crise environnementale, c'est une révolution géologique d'origine humaine" [la traducción es mía].

2 El Grupo de Trabajo del Antropoceno (AWG, por sus siglas en inglés) ha definido el Antropoceno como una época. En la clasificación estratigráfica geológica, se considera que una era tiene un estatus superior. Por ello, en todos los artículos de la sección "Saberes y razones" de este número de Desacatos adoptamos el término "época" para hacer referencia al Antropoceno: "el Antropoceno ha sido traducido en los medios de comunicación populares como la 'edad de los humanos'. propondría que fuera la 'época de los seres humanos'. También podríamos decir 'época de los humanos', pero 'seres humanos' me parece una traducción más precisa" [comunicación personal con Helmuth Trischler, 23 de marzo de 2017]. 
se ha convertido en un factor del sistema global Tierra. Una hipótesis que, de resultar probada, tiene una enorme gama de implicaciones (Leinfelder, 2013).

La tesis central del Antropoceno parte de que la humanidad ha afectado a la naturaleza al grado de ser responsable del nuevo estrato en el récord geológico. La periodización relativa a su origen ha sido causa de múltiples discusiones, relacionadas en particular con la identificación de su comienzo. Se había considerado que todo empezó con la Revolución industrial, hace más de 200 años, y que su evolución se magnificó a partir de la Segunda Guerra Mundial, en lo que se denominó "la gran aceleración”, que incluyó elementos como los siguientes:

- crecimiento muy dinámico de la población

- urbanización desbordada

- desarrollo intenso de la infraestructura

- consumo en incremento constante

- explotación desenfrenada de recursos

- intensificación y diversificación de la pobreza

- pérdida de la biodiversidad

Las discusiones estaban en proceso de llegar a acuerdos cuando el presente número de Desacatos estaba en preparación. Tras siete años de deliberaciones, en el 35 Congreso Internacional de Geología, celebrado en agosto de 2016 en Ciudad del Cabo, Sudáfrica, los miembros del Grupo de Trabajo del Antropoceno (AWG, por sus siglas en inglés) ${ }^{3}$ votaron de manera unánime para reconocer oficialmente al Antropoceno como una época geológica en la historia terrestre. De acuerdo con los miembros de este grupo interdisciplinario, Antropoceno es paralelo al Pleistoceno y el Holoceno, y se ubica dentro del periodo Cuaternario. Se fechó su principio en ca. 1950, por factores como las pruebas de bombas nucleares, la agricultura industrializada, el cambio climático y la proliferación del plástico. ${ }^{4} \mathrm{El}$ denominado golden spike, ${ }^{5}$ que se puede traducir como "clavo dorado", que separa al Antropoceno o "epóca de los seres humanos" del resto de la historia, comienza a mediados del siglo XX, cuando la llamada "gran aceleración” empezó en la Tierra y los impactos humanos en el planeta se intensificaron e hicieron cada vez más globales.

Las transformaciones experimentadas no presentan visos de cambiar de dirección, disminuir su gravedad o aligerarse, lo que presagia cambios a largo plazo y en todo el globo terrestre. Se ha reconocido que se está creando un nuevo modo de desarrollo planetario, para el cual se han acuñado varios términos que acompañan al de Antropoceno, como el de "neurogeología" (Schwägerl, 2013), o que lo sustituyen por otros, como Capitaloceno, "fase interna de la mundialización capitalista, que no es sinónimo de fin de la historia" (Altvater, 2014: 5). ${ }^{6}$

El trabajo seminal que originó esta revolución del pensamiento en los albores del siglo XXI fue publicado en el año 2000, en el número 41 del Global Change Newsletter del International Geosphere-Biosphere Programme (IGBP). Abarca sólo dos páginas, con el título llano y directo de "El 'Antropoceno"” - "The "Anthropocene"- - Sus autores, el holandés Paul J. Crutzen y el estadounidense

3 El Awg depende de la Subcomisión de Estratigrafía del Cuaternario (SQS, por sus siglas en inglés) y la Comisión Internacional de Estratigrafía (Ics, por sus siglas en inglés) dentro de la Unión Internacional de Ciencias Geológicas (IUGS, por sus siglas en inglés) (sQS, 2016).

$4 \quad$ Varios autores de este número de Desacatos debieron incorporar cambios a sus textos para considerar la decisión del AWG, dada a conocer el 29 de agosto en el periódico inglés The Guardian (Carrington, 2016).

$5 \quad$ Se define como una señal distintiva y medible de la presencia humana en el registro geológico, que permanece por un periodo extremadamente prolongado (Schwägerl, 2013: 31).

6 Una interesante confrontación entre ambos términos, Antropoceno y Capitaloceno, se encuentra en Moore (2016) y en los artículos de Ulloa y Thomas, en este número de Desacatos. 
Eugene F. Stoermer, ${ }^{7}$ parten de un breve recuento de los científicos que desde mediados del siglo XIX reconocieron que las actividades del ser humano se habían convertido en una fuerza geológica y morfológica creciente en la Tierra y la atmósfera. Hablan de geólogos, paleontólogos, matemáticos y científicos en general, provenientes de diferentes partes del mundo occidental, como George Perkins Marsh, de Estados Unidos; Antonio Stoppani, de Italia; Vladimir I. Vernadsky, de Rusia, y Pierre Teilhard de Chardin y Édouard Le Roy, de Francia. Al considerar los grandes y crecientes efectos globales, así como el impacto de las actividades humanas en la ecología y la geología, que se han hecho cada vez más evidentes en particular durante los últimos dos siglos — que coinciden con la invención y masificación del uso de la máquina de vapor de James Watt, en 1784-, proponen usar el término "Antropoceno" para denominar la época geológica contemporánea. Advierten que por primera vez la humanidad es una fuerza geológica y que así continuará por muchos milenios, millones de años quizá (Crutzen y Stoermer, 2000). ${ }^{8}$

El planteamiento del Antropoceno ha tomado posición rápidamente en los círculos europeos y estadounidenses desde hace más de una década. Numerosas reuniones, congresos, publicaciones, cursos e incluso revistas dedicadas exclusivamente a ello han surgido y tenido gran aceptación. ${ }^{9}$ Se trata de una discusión que incursionó en el campo de las ciencias sociales recientemente y que apenas lo ha hecho en el sur del planeta. ${ }^{10}$

Tres experiencias me permitieron tener contacto con esta nueva propuesta:

a) Las discusiones sobre el Antropoceno que sostuve con los directivos y profesores visitantes en el Rachel Carson Center for Development and Society (RCC), tema que ha sido parte de sus intereses y sobre el cual el RCC ha lanzado publicaciones diversas. ${ }^{11} \mathrm{~A}$ lo que se sumó la visita a la exposición conjunta del RCC y el Deutsches Museum, en la ciudad de Múnich, que llevó como título Welcome to the Anthropocene. The Earth in our Hands - Bienvenidos al Antropoceno. La Tierra en nuestras manos- y cuyo principal objetivo fue, desde su concepción,

7 Crutzen (1933-) es Premio Nobel de Química 1995, junto con el mexicano Mario Molina y el estadounidense Frank S. Rowland; vicepresidente del IGBP e investigador del Instituto Max Planck de Química. Stoermer (1934-2012) fue biólogobotánico e investigador de la Universidad de Michigan, así como miembro de numerosos consejos académicos especializados en sus temas. A él se le atribuye el haber lanzado la idea original del concepto en la década de 1980.

8 Especialistas como Clive Hamilton y Jacques Grinevald (2015) argumentan que no existen precursores de la noción de Antropoceno, dado que su aparición en escena es producto de discusiones interdisciplinarias que tratan de entender, no la Tierra, sino el Sistema Tierra: el Antropoceno representa una ruptura radical respecto a las ideas evolucionistas en la historia de la humanidad y la historia de la Tierra.

9 Dos de las mejores hasta el momento, ambas autodefinidas como transdisciplinarias o interdisciplinarias, son Anthropocene y The Anthropocene Review, que surgieron en 2013 y 2014, respectivamente.

10 La revista The Anthropocene Review es la que incluye el mayor número de artículos relacionados con ciencias sociales y humanidades; no abstante, los temas y autores latinoamericanos son casi inexistentes tanto en The Anthropocene Review como en Anthropocene. Esperamos que bajo la conducción de Georgina Endfield, editora de The Anthropocene Review, apreciada colega geógrafa y especialista en temas de clima en el México colonial, se tome ese rumbo y se incorporen miradas provenientes de esta región.

11 El RCc es un centro para la investigación y la educación en temas ambientales y sociales, fundado en 2009 como una iniciativa conjunta entre la Universidad Ludwig-Maximilians de Múnich y el Deutsches Museum, con el apoyo del Ministerio Federal para la Educación y la Investigación de Alemania. Agradezco de nuevo la gentil invitación del director del RCc, Christof Mauch, para que realizara allí una estancia en julio de 2015, dentro de mi año sabático, y experimentara los beneficios de las fantásticas condiciones que ese centro ofrece e incursionara de manera más directa en el tema del Antropoceno. 

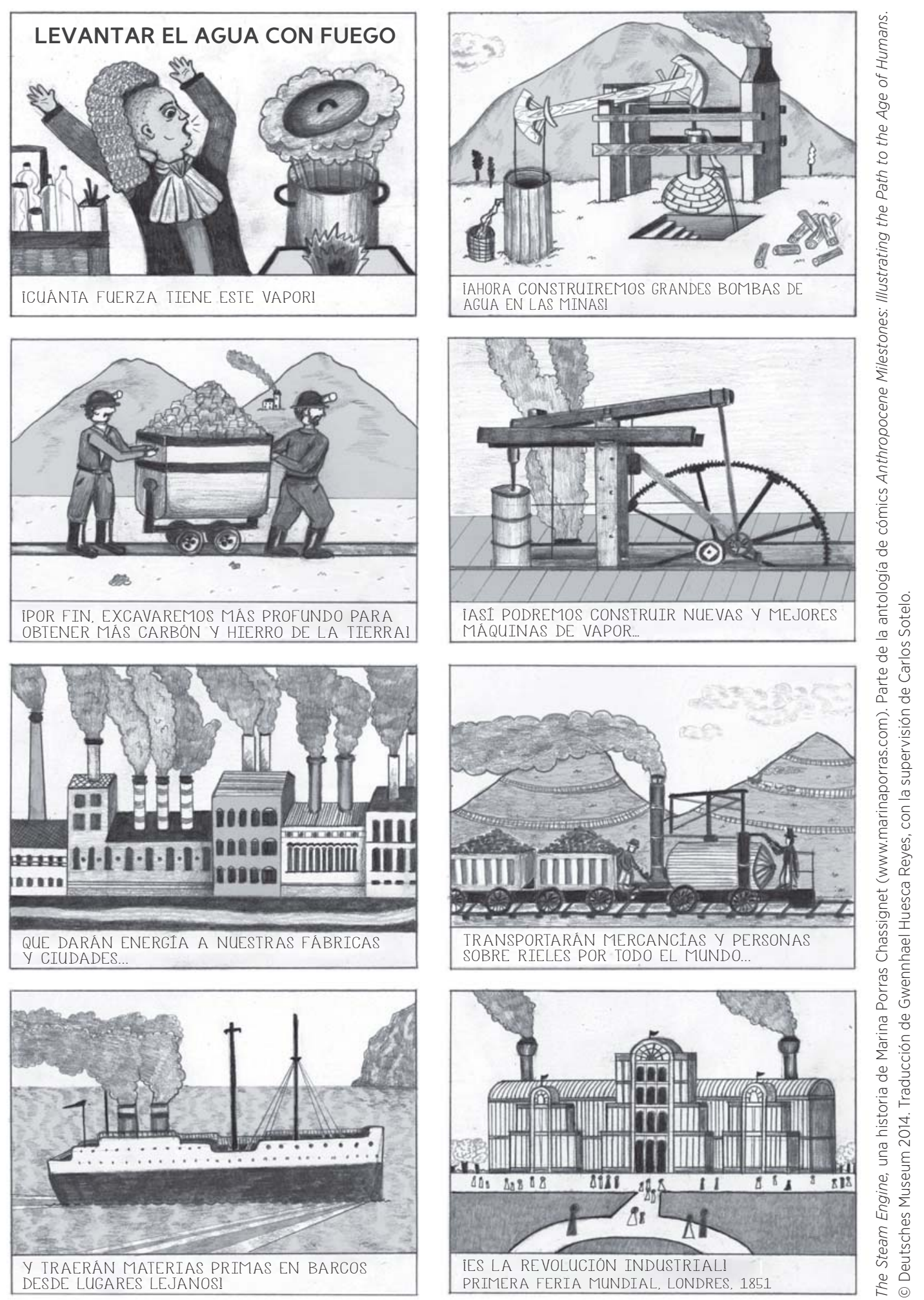
traducir un concepto teórico y sus principales argumentos en una exhibición museográfica para todo el público interesado (Möllers, 2013). ${ }^{12}$

b) La participación en el Coloquio “¿Cómo pensar el Antropoceno? Antropólogos, filósofos y sociólogos frente al cambio climático", producto de una convocatoria conjunta entre Philippe Descola, del Collège de France, y Catherine Larrère, de la Fondation de l'Écologie Politique, que tuvo lugar en París, en noviembre de $2015 .{ }^{13}$

c) La asistencia a la segunda versión del Anthropocene Campus, organizado conjuntamente por la Haus der Kulturen der Welte y el Max Planck Institute for the History of Science, como parte de "The Anthropocene Curriculum Project" (ACP). ${ }^{14}$

En el Coloquio “¿Cómo pensar el Antropoceno?”, llamó mi atención la casi total ausencia de latinoamericanos en las discusiones. Más allá de participantes destacados que, como Philippe Descola, han trabajado en la región, del total de más de medio centenar de ponentes y conferencistas, sólo tres éramos latinoamericanos. ${ }^{15}$ Durante el Coloquio le fui dando forma a la idea de introducir la discusión sobre el Antropoceno en nuestros países. Invité a Descola, quien, con gran generosidad, aceptó de inmediato y me entregó la conferencia que acababa de dictar, "Humain, trop humain?". ${ }^{16}$ Animé también a Astrid Ulloa para que ofreciera una perspectiva sudamericana a partir de su experiencia como antropóloga con población vernácula y movimientos indígenas en Colombia. Lo mismo hice con Franz Mauelshagen, colega historiador alemán, encargado del proyecto "The Anthropocene: Reuniting Earth History with Human History" en el Institute for Advanced Sustainability Studies (IASS), de Potsdam, Alemania.

Posteriormente, convoqué a Helmuth Trischler, a quien conocí durante mi estancia en el RCC, pues es su codirector y también director de investigación del Deutsches Museum, en el que estuvo a cargo del equipo de trabajo que concibió, montó y cuidó la exposición Welcome to the Anthropocene. ${ }^{17}$ Especialista con varias publicaciones sobre el tema, aceptó y contribuyó con una amplia introducción al tema, dedicada en particular a los lectores del mundo hispanohablante.

12 La exposición estuvo vigente de diciembre de 2014 a septiembre de 2016. Fue la primera gran exhibición sobre el tema, a partir de la presentación de asuntos seleccionados ad hoc, con artefactos y tecnología relacionados con urbanización, movilidad, naturaleza, evolución y alimentos. Un espléndido catálogo da testimonio de todo ello (Möllers, Schwägerl y Trischler, 2015). Véase también Robin et al. (2014).

13 Este magno evento reunió a más de medio centenar de ponentes y conferencistas, especialistas en las disciplinas que mencionaba la convocatoria. La asistencia rondó entre las 700 y 800 personas. Toda la información y la videograbación de las presentaciones, incluso las dictadas por los dos organizadores, y aquella con abrumadora presencia de estudiantes a cargo de Bruno Latour, están disponibles en: $<$ https://anthropocene2015.wordpress.com/> y <http://www. fondationecolo.org/l-anthropocene/video>. Consultados el 18 enero de 2017

14 Entre 2013 y 2014, se desarrolló el Anthropocene Project, en cuya apertura, en enero de 2013, participó el paleobiólogo Jan Zalasiewic, gran impulsor del Antropoceno, bajo el auspicio de esas dos instituciones, además del Institute for Advanced Sustainability Studies y el Rcc. De él se derivó el ACP, cuyo principal objetivo es reunir académicos, artistas y miembros de la sociedad civil de todo el mundo para constituir un cuerpo dinámico de conocimiento experimental y autorreflexivo para discutir y enfrentar los retos que se presentan en el siglo XXI. El ACP se reunió por primera vez en noviembre de 2014 y por segunda vez en abril de 2016, en los denominados Anthropocene Campus 1 (ACl) y Anthropocene Campus 2 (Ac2), respectivamente. Disponible en: <http:// www.anthropocene-curriculum.org/>. Consultado el 18 enero de 2017

15 Astrid Ulloa y Germán Palacio, de Colombia, y yo, de México. Los demás participantes procedían de Europa -Alemania, Francia, Inglaterra, Italia, Portugal, Suecia, Suiza-, Norteamérica -Estados Unidos y Canadá- y Australia.

16 Que se reproduce en este número de Desacatos, traducida al español.

17 A la que alude en su texto, publicado también aquí. 
Contaba ya con cuatro especialistas para preparar este número sobre "Cambio climático y Antropoceno": dos antropólogos y dos historiadores, tres europeos y una latinoamericana. Requería una combinación que permitiera una mejor equidad continental. Gracias a la sugerencia de Mauelshagen, conocí e invité a Julia Adeney Thomas, historiadora económica estadounidense, quien aportó el excelente trabajo que terminó por completar el cuadro para ofrecer un panorama bien integrado sobre el tema.

Por último, el proyecto se redondeó con mi asistencia en calidad de observadora, gracias a la invitación del IASS, a la segunda edición del ACP, "The Tecnosphere Issue”, celebrado en Berlín, en abril de 2016. ${ }^{18}$ Como advirtieron sus organizadores, el objetivo era arrojar luz sobre esta esfera artificial y desafiarnos a comprender, describir y definir de manera más consciente un siglo XXI en el que las fuerzas de la ley de la industria, la tecnología, la cultura y la vida actúan en correspondencia con los límites biofísicos y las posibilidades de nuestro planeta. ${ }^{19}$

Éste no es el lugar para reproducir la enorme cantidad de discusiones que se produjeron alrededor del origen y evolución de los conceptos de Antropoceno, tecnosfera y coevolución, incluso de naturaleza y cultura. Lo que quiero destacar es que ello permitió confirmar la ausencia casi total de la perspectiva de una gran región del sur del planeta, y por lo tanto, la necesidad de ampliar la discusión de este lado del Atlántico y en las zonas tórridas. También es momento de incorporar las pocas experiencias que existen al respecto, como las resultantes del Colóquio Internacional Os Mil Nomes de Gaia: do Antropoceno à Idade da Terra, celebrado en Río de Janeiro, Brasil, en septiembre de 2014, con ponencias de excelente factura. ${ }^{20}$

Durante una sesión de reflexión, al finalizar la extensa y concurrida reunión de abril en Berlín, se recordó que la idea original era incubar y fomentar un marco interdisciplinario para coaprender, coproducir, explorar y establecer nuevas formas de conocimiento relacionadas con el Antropoceno. En esa ocasión, ofrecí de manera explícita contribuir a esa discusión global a partir de varias iniciativas cuyo objetivo sería incorporar la mirada del sur en esta nueva conceptualización, entre ellas, la publicación de las contribuciones en un medio conocido, reconocido y bien posicionado en el campo académico hispanohablante, Desacatos. Revista de Ciencias Sociales. ${ }^{21}$

El resultado muestra que hemos privilegiado la perspectiva de historiadores y antropólogos, lo que responde a la coincidencia de que las tesis del Antropoceno demandan la transdisciplinariedad o interdisciplinariedad, pero a la vez reconocemos que el diálogo entre esas dos disciplinas en particular resulta ineludible, más aún en lo tocante a los temas que aquí nos ocupan. A ello se suma, a la

A este concurrido y singular evento, que tuvo como sede la fantástica Casa de las Culturas del Mundo (HKW, por sus siglas en alemán), acudieron cerca de 200 personas, entre participantes e instructores; se desarrollaron diez seminarios, tres public evenings sobre "Tecnosfera y conocimiento" y otras actividades literarias y musicales. Entre los participantes e instructores, Latinoamérica estuvo prácticamente ausente. Véase HKW (2016).

19 Véase The Anthropocene Curriculum (2016).

20 De este coloquio tuve conocimiento gracias a Astrid Ulloa. Fue organizado por el Departamento de Filosofía de la Pontificia Universidad Católica y el Programa de Posgrado en Antropología Social del Museo Nacional-Universidad Federal de Río de Janeiro. Estuvieron presentes especialistas de la talla de Bruno Latour, de Francia; Donna Haraway, de Estados Unidos; Clive Hamilton, de Australia; Lesley Green, de Sudáfrica; Alf Hornborg, de Suecia, y Eduardo Viveiros do Castro, de Brasil. Contó con una mayoritaria participación de brasileños de varias especialidades. Disponible en línea: <https://osmilnomesdegaia.eco.br>. El antropólogo Renzo Taddei, de la Universidad Estatal de São Paulo, con quien hemos colaborado en ocasiones anteriores, estuvo también en el AC2. Aun así, la presencia de Latinoamérica ha sido sumamente reducida.

21 En principio, mi compromiso es dar a conocer este número sobre "Cambio climático y Antropoceno" en la página del ACP, la cual se sigue alimentando con los productos o iniciativas en curso relacionadas con el tema. Véase The Anthropocene Curriculum (2015). 
vez que enriquece, el hecho de que los cinco autores que ofrecen discusiones en este número de Desacatos incorporan un diálogo con otras especialidades: ciencia política, sociología, filosofía, economía, como se pretendía que ocurriera y en efecto ocurrió en el Coloquio “¿Cómo pensar el Antropoceno?”.

El objetivo central de presentar este tema es motivar su discusión en México y el resto de Latinoamérica, donde su conocimiento todavía es incipiente, para debatir, coincidir o bien disentir, pero hacerlo con una mirada desde el sur. O bien, para "tropicalizarlo", como lo hace Astrid Ulloa cuando se refiere al concepto de Capitaloceno, al que relaciona con el extractivismo en el caso colombiano.

En la literatura sobre Antropoceno hay en realidad muy pocas publicaciones en español, que sean de, desde, y sobre todo, para Latinoamérica. La mayor parte de esa bibliografía y de las reflexiones está en inglés. Es del Norte para el Norte, con muy pocas excepciones. ${ }^{22}$ Urge entonces llevar a cabo un diálogo Norte-Sur sobre estos temas, entre el Norte-global y el Sur-global.

Así como se lo propueso Amelia Moore (2015) en el compendio que coordinó, publicado por Environment and Society, con la publicación de este número de Desacatos pretendo que el diálogo alrededor del Antropoceno, sus críticas y aportaciones, sirvan no sólo como "alimento para el pensamiento" — food for thought—, sino como "combustible para el pensamiento" - fuel for thought - y a la vez, como catalizador de un mayor compromiso con el planeta, que estimule el debate sobre el Antropoceno y sus vinculaciones con el cambio climático, incluso con la reducción de riesgos de desastres. ${ }^{23} \mathrm{D}$

22 En abril de 2016, tuve la fortuna de asistir a la conferencia de Federico Navarrete, del Instituto de Investigaciones Históricas de la Universidad Nacional Autónoma de México, titulada "La cosmohistoria o cómo pensar la globalización en el Antropoceno", en el marco de la Conferencia Internacional del Colegio Internacional de Graduados "Entre espacios", iniciativa conjunta de instituciones mexicanas y alemanas. Véase LAI (2016). Las interesantes propuestas de Navarrete, entre las que destaca la de la cosmohistoria como un intermedio entre Antropoceno y Capitaloceno, deberán alimentar el diálogo Norte-Sur.

23 Hago referencia a la discusión entre estos tres elementos en el trabajo resultante de mi participación en el Coloquio "¿Cómo pensar el Antropoceno?” (García Acosta, en prensa).

\section{Bibliografía}

Altvater, Elmar, 2014, "El capital y el Capitaloceno”, en Mundo Siglo XXI, núm. 33, vol. IX, pp. 5-15.

Bonneuil, Christophe y Jean-Baptiste Fressoz, 2013, L'Evénement Anthropocène. La Terre, I'histoire et nous, Le Seuil (Anthropocène), París.

Crutzen, Paul J. y Eugene F. Stoermer, 2000, "The 'Anthropocene'”, en Global Change Newsletter, núm. 41, pp. 17-18.

García Acosta, Virginia, en prensa, "Unnatural Disasters and the Anthropocene: Lessons Learnt from Anthropological and Historical

Perspectives", en Rémi Beau y Catherine Larrère (eds.), How to Think the Anthropocene? Anthropologists, Philosophers, and Sociologists facing Climate Change, Fondation de l'Écologie Politique, París.

Hamilton, Clive y Jacques Grinevald, 2015, “Was the Anthropocene Anticipated?”, en The Anthropocene Review, vol. 2, núm. 1, pp. 59-72.

Leinfelder, Reinhold, 2013, "Assuming Responsibility for the Anthropocene: Challenges and Opportunities in Education", en Helmuth Trischler (ed.), Anthropocene. Envisioning the Future of the Age of Humans, Rachel Carson Center for Environment and Society, Múnich, pp. 9-28.

Moore, Amelia (coord.), 2015, "The Anthropocene. A Critical Exploration”, en Environment and Society: Advances in Research, vol. 6, núm. 1, pp. 1-3.

Moore, Jason W. (ed.), 2016, Anthropocene or Capitalocene? Nature, History, and the Crisis of Capitalism, PM Press, Oakland.

Möllers, Nina, 2013, "Cur(at)ing the Planet. How to Exhibit the Anthropocene and Why", en Helmuth Trischler (ed.), Anthropocene Envisioning the Future of the Age of Humans, Rachel Carson Center for Environment and Society, Múnich, pp. 57-66. 
Möllers, Nina, Christian Schwägerl y Helmuth Trischler (eds.), 2015, Welcome to the Anthropocene: The Earth in Our Hands, Deutsches Museum Verlag, Múnich.

Robin, Libby et al., 2014, "Three Galleries of the Anthropocene”, en The Anthropocene Review, vol. 1, núm. 3, pp. $207-224$.

Schwägerl, Christian, 2013, "Neurogeology: The Anthropocene's Inspirational Power", en Helmuth Trischler (ed.), en Anthropocene Envisioning the Future of the Age of Humans, Rachel Carson Center for Environment and Society, Múnich, pp. 29-38.

\section{Sitios de internet}

Anthropocene Curriculum, The, 2015. Disponible en línea: <http://www.anthropocene-curriculum.org/>. Consultado el 18 enero de 2017.

_, The, 2016, “Campus 2016. The Technosphere Issue”. Disponible en línea: <http://www.anthropocene-curriculum.org/pages/root/ campus-2016/>. Consultado el 18 enero de 2017.

Carrington, Damian, 2016, "The Anthropocene Epoch: Scientists Declare Dawn of Human-Influenced Age", en The Guardian, 29 de agosto. Disponible en línea: <https://www.theguardian.com/environment/2016/aug/29/declare-anthropocene-epoch-experts-urge-geological-congress-human-impact-earth>. Consultado el 1 de noviembre de 2016

Comment penser l'Anthropocène? Anthropologues, philosophes et sociologues face au changement climatique, 2015. Disponible en línea: <https://anthropocene2015.wordpress.com/>. Consultado el 18 enero de 2017.

Fondation de l'Écologie Politique, 2015, "Comment penser l'Anthropocène? Videos". Disponible en línea: <http://www.fondationecolo.org/ I-anthropocene/video>. Consultado el 18 enero de 2017.

Haus der Kulturen der Welt (HKw), 2016, “Campus 2016: The Technosphere Issue”. Disponible en línea: <https://www.hkw.de/en/programm/ projekte/2015/curriculum_campus_technosphere/campus_the_technosphere_issue.php>. Consultado el 18 enero de 2017.

Lateinamerika-Institut, Freie Universität Berlin (LAI), 2016, "Zwischen Räumen”. Disponible en línea: <http://www.lai.fu-berlin.de/entre-espacios/>. Os Mil Nomes de Gaia: do Antropoceno à Idade da Terra, 2015. Disponible en línea: <https://osmilnomesdegaia.eco.br>.

Subcommission on Quaternary Stratigraphy (SQS), 2016, "Working Group on the Anthropocene”. Disponible en línea: <http://quaternary. stratigraphy.org/workinggroups/anthropocene/>. Consultado el 1 de noviembre de 2016. 